\title{
Erratum: Droplet pattern and condensation gradient around a humidity sink [Phys. Rev. E 89, 012402 (2014)]
}

\author{
J. Guadarrama-Cetina, R. D. Narhe, D. A. Beysens, and W. González-Viñas
}

(Received 12 February 2020; accepted 14 February 2020; published 2 March 2020)

DOI: 10.1103/PhysRevE.101.039901

Corrections, which have no consequences on the conclusions of the paper, have to be considered in some places to make reading and understanding easier.

In the abstract (line 8), the exponent of the growth rate of the salty drop $R$ should read $\frac{1}{5}$ instead of 5 .

On p. 3 (Sec. II E), values of $\frac{d \sigma}{d T}$ and Marangoni number (Ma) are incorrect and should read $\frac{d \sigma}{d T} \approx-1.5 \times 10^{-4} \mathrm{~N} \mathrm{~m}^{-1} \mathrm{~K}^{-1}$, and, Ma should read $\simeq 4 \times 10^{2} \Delta T$, respectively. With the critical Marangoni number $\mathrm{Ma}_{c} \simeq 80$, thermocapillary flows can start for temperature differences as small as $0.2 \mathrm{~K}$ (instead of $20 \mathrm{mK}$ as stated in the paper). This value also ensures an efficient heat transfer from the drop interface to the condensing surface and validates a three-dimensional hyperbolic water pressure profile around the drop.

The caption for Fig. 1, should read "Type I (a) and (b) and type II (c)-(f) snapshots of the experiment. (a) Time $t=0$. (b) $t=2000 \mathrm{~s}$ with the breath figure (BF) around the region of inhibited condensation. (c) At $t=0 \mathrm{~s}$, a NaCl crystal is placed on the substrate. (d) Even if the crystal has not been totally dissolved, BF condensation is visible at $t=50 \mathrm{~s}$. (e) BF is clearly visible on the observable area $(t=125 \mathrm{~s})$. A small bubble of trapped air is visible. (f) At $t=1000 \mathrm{~s}$, the stage of the system is comparable to (b)." The 0 subscript of $R$ in Fig. 1(e) should not appear.

On p. 4 (Sec. III, last paragraph of the left column), “...In Fig. 1(e), the process of completely dissolving the crystal lasts about $250 \mathrm{~s}$, and the drop radius is then $R_{0}=(243 \pm 12) \mu \mathrm{m}$ corresponding to the salt saturation as for the drop in the type I experiment ...." should read as: "...The process of completely dissolving the crystal lasts about $250 \mathrm{~s}$, and the drop radius is then $R_{0}=(310 \pm 12) \mu \mathrm{m}$ corresponding to the salt saturation as for the drop in the type I experiment at the initial time ...."

On p. 4 (Sec. IV, end of the second paragraph), "The drop radius fits well with Eq. (19) with $R_{0}$ as $310.9 \mu \mathrm{m}$, giving $a=4.98 \pm 0.01$ and $\tau=(300 \pm 2)$ s." should read: "The drop radius fits well with Eq. (19) with $R_{0}$ as $310.2 \mu \mathrm{m}$, giving $a=5.00 \pm 0.01$ and $\tau=(262 \pm 2)$ s."

On p. 4 (Sec. IV, last paragraph), “...On a purely hydrophilic substrate, nucleation corresponds to the saturation pressure $p_{s}\left(=1.2 \mathrm{kPa}\right.$ for $\left.T_{s}=10^{\circ} \mathrm{C}\right)$. On the hydrophobic substrate used here, a temperature difference of $\left.\simeq 1.5^{\circ} \mathrm{C}\right)$ with respect to room temperature is needed to observe nucleation of water, corresponding to a supersaturation $\Delta p(\simeq 0.25 \mathrm{kPa})$ [27]. This corresponds to the water vapor pressure $p_{0}=p_{s}+\Delta p(\simeq 1.45 \mathrm{kPa}) \ldots$. . should read: “...On a purely hydrophilic substrate, nucleation corresponds to the saturation pressure of $p_{\infty}\left(=2.8 \mathrm{kPa}\right.$ for room temperature of $\left.T=23^{\circ} \mathrm{C}\right)$. On the hydrophobic substrate used here, a temperature difference of $\simeq 1.5^{\circ} \mathrm{C}$ with respect to room temperature is needed to observe nucleation of water, corresponding to a supersaturation $\Delta p(\simeq 0.25 \mathrm{kPa})$. This corresponds to the water vapor pressure $p_{0}=p_{\infty}-\Delta p$ $(\simeq 2.55 \mathrm{kPa}) \ldots .$.

In Fig. 3, the mark corresponding to $p_{0}$ is misplaced and should take a value of $2.55 \mathrm{kPa}$.

On p. 6 (Sec. VI, last paragraph), “...The expected values are $C=\frac{p_{\infty}-p_{s}}{p_{\infty}-p_{0}}=1.185$ and $D=\frac{p_{s}-p_{s 0}}{p_{\infty}-p_{0}}=0.21 \ldots$. .. should read: "...The expected values are $C=\frac{p_{\infty}-p_{s}}{p_{\infty}-p_{0}}=6.36$ and $D=\frac{p_{s}-p_{s 0}}{p_{\infty}-p_{0}}=1.24 \ldots .$. " Also "...They differ, however, for $C \approx 0.5$ (type I) and $\approx 1.2$ (type II), also in disagreement with the expected values ...." should read as: “...They differ, however, for $C \approx 0.5$ (type I) and $\approx 1.2$ (type II), also in disagreement for type I with the expected value. A good agreement between expected values and type II is however found ...."

On p. 8 [paragraph after Eq. (36)], "The data fits the above variation reasonably well, with $E=0.028 \pm 0.002$ and $F=$ $0.9 \pm 0.01$ (type I, one standard deviation), $E=0.044 \pm 0.001$ and $F=1.16 \pm 0.03$ (type II, one standard deviation). The $F$ value also agrees well between experiments types I and II, and the expected value for $\frac{p_{\infty}-p_{0}}{p_{\infty}-p_{s}} \simeq 0.71 \ldots$.." should read: "The data fit the above variation reasonably well with $E=0.025 \pm 0.002$ and $F=1.1 \pm 0.01$ (type I, one standard deviation), $E=$ $0.051 \pm 0.001$ and $F=0.86 \pm 0.03$ (type II, one standard deviation). The $F$ value agrees well between experiments types I and II but somewhat differs from the expected value of $\frac{p_{\infty}-p_{0}}{p_{\infty}-p_{s}} \simeq 0.16 \ldots . "$

On p. 8 [row above Eq. (42)], "Nucleation is not possible between drops ..." should read "Nucleation is possible between drops ..."

[27] J. Guadarrama-Cetina and W. González-Viñas, Phys. Rev. E 87, 054401 (2013). 